\title{
NOTE ON A TOPOLOGY OF A DUAL SPACE
}

\section{MORIKUNI GOTO ${ }^{1}$}

1. Introduction. ${ }^{2}$ Let $G$ be a locally compact group, and let $L$ be a Lie group. Let us denote by $D$ the set of all continuous homomorphisms from $G$ into $L$. By introducing the so-called compact-open topology in $D, D$ becomes a complete uniform space. We shall call the uniform space $D$ the dual space of $G$ with respect to $L$ in this paper.

When $G$ is abelian and $L$ is the group of rotations in the euclidean plane, $D$ coincides with the character group in the sense of Pontrjagin, and it is well known that $D$ is also locally compact. Here we would like to generalize the proposition to the nonabelian case. The purpose of this note is to prove the following two theorems:

Theorem 1. Let $G$ be a locally compact group, and $L$ a compact Lie group. Then the dual space $D$ is locally compact.

THEOREM 2. Let $G$ be a locally compact group and L a Lie group. If there is a compact generating system in $G$, then the dual space $D$ is locally compact.

REMARK. If $G$ is an infinitely generated free group with the discrete topology, and if $L$ is a noncompact Lie group, then $D$ is homeomorphic with an infinite product of copies of $L$ and accordingly $D$ is not locally compact.

2. Preliminaries. ${ }^{2}$ Let $M$ be a set and $H$ a topological group. Let us denote by $(M \rightarrow H)$ the topological space composed of all functions from $M$ into $H$ relative to the product topology (= finite-open topology). By defining the multiplication: $(f g)(x)=f(x) g(x)$ for $f, g$ $\in(M \rightarrow H)$ and $x \in M,(M \rightarrow H)$ becomes a topological group. When $M$ is a topological space, we consider a subset (subgroup) $[M \rightarrow H]$ of $(M \rightarrow H)$ composed of all continuous functions. For a compact subset $C$ of $M$ and a neighborhood $E$ of the identity in $H$ we define a subset

$$
(C, E)=\{f \mid f \in[M \rightarrow H] \text { and } f(C) \subset E\} \text { of }[M \rightarrow H] .
$$

It is easy to see that the set of all possible $(C, E)$ 's forms a base for a neighborhood system of the identity of a topological group. After

Received by the editors December 1, 1959.

1 The work of this author was supported in part by NONR 55130.

${ }^{2}$ For the first two sections please refer to the books listed in the end of the paper. 
this $[M \rightarrow H]$ denotes the topological group thus topologized. Clearly the identity mapping brings $[M \rightarrow H]$ continuously into $(M \rightarrow H)$.

Let us assume that $M$ is a topological group. Let $D^{\prime}$ be the set of all homomorphisms from $M$ into $H$. We define $D$ by $D=D^{\prime} \cap[M \rightarrow H]$. Since an element $f$ of $D^{\prime}$ is characterized by the property $f(x) f(y)$ $=f(x y)$ for any pair $x, y$ of $M, D^{\prime}$ is a closed subspace of $(M \rightarrow H)$. $D$ is closed in $[M \rightarrow H]$ similarly. When $H$ is a complete topological group $(M \rightarrow H)$ and $[M \rightarrow H]$ are known to be complete, and so $D^{\prime}$ and $D$ are complete. Hence $D^{\prime}$ and $D$ are complete uniform spaces. It is to be noted that when $H$ is locally compact $f(x)$ is a jointly continuous function from $[M \rightarrow H] \times M$ into $H$.

Proposition 1. Let $G$ and $L$ be locally compact groups. Then (1) $(G \rightarrow L)$ and $[G \rightarrow L]$ are complete topological groups, and $D^{\prime}$ and $D$ are closed subspaces of $(G \rightarrow L)$ and $[G \rightarrow L]$ respectively. (2) For an $f$ in $[G \rightarrow L] f(x)$ is jointly continuous from $[G \rightarrow L] \times G$ into $L$. (3) When $L$ is compact, $D^{\prime}$ is compact.

Notations. We shall write 1 for the identity of the group in question. By a nucleus of a locally compact group we mean an open symmetric neighborhood of 1 , whose closure is compact. Let $G$ and $L$ be locally compact groups, and $D$ the dual space of $G$ with respect to $L$. Let $f$ be in $D$. For a compact subset $C$ of $G$ and a nucleus $E$ of $L$ we put

$$
(f ; C, E)=\left\{g \mid g \in D \text { and }\left(f^{-1} g\right)(C) \subset E\right\} .
$$

The set of all possible $(f ; C, E)$ 's of course forms a base for the neighborhood system of $f$ in $D$.

Let $L$ be a Lie group of dimension $r$. Let us introduce a canonical system of coordinates of the first kind in a suitable nucleus of $L$. By changing the scale if necessary, we may identify a nucleus $E$ with an open euclidean sphere of radius 2: for $a$ in $E$, there corresponds coordinates $\left(a_{1}, \cdots, a_{r}\right)$ so that $\|a\|=\left(a_{1}^{2}+\cdots+a_{r}^{2}\right)^{1 / 2}<2$. We denote $S(\delta)=\{a \mid\|a\|<\delta\}$ for $0<\delta \leqq 2$. By the definition of a canonical coordinate system of the first kind, $t a=\left(t a_{1}, \cdots, t a_{r}\right)$ forms a local one-parameter subgroup for $-2 /\|a\|<t<2 /\|a\|$, and if $j$ is a positive integer less than $2 /\|a\|$, then $a^{j}=j a$.

Proposition 2. Let $m$ be a positive integer. Then an element a of $L$ is contained in the sphere $S(1 / m)$ if and only if $a, a^{2}, \cdots, a^{m} \in S(1)$.

Proof. If $a \in S(1 / m)$, namely if $\|a\|<1 / m$, then for $j \leqq m, j<1 /\|a\|$ $<2 /\|a\|$, and so $a^{j}=j a$ and $\left\|a^{j}\right\|=j\|a\|<1$.

Conversely if $a$ is in $S(1)$ but not in $S(1 / m)$, namely if $1 / m \leqq\|a\|$ 
$<1$, and if $j$ is the first integer so that $1 \leqq j\|a\|$, then $j \leqq m$ and $j\|a\|<2$, whence $a^{j}=j a \notin S(1)$.

3. Proof of Theorem 1. Let $G$ be a locally compact group, and $L$ a compact Lie group. We retain the notations in 2 . We know that $(G \rightarrow L)$ is compact, and the identity mapping $I$ is continuous and one-to-one from $D$ into $(G \rightarrow L)$. Let $f$ be an element of $D$. Since $f$ is continuous we can find a nucleus $V$ of $G$ so that $f(V) \subset S(1 / 2)$. Take a nucleus $E$ of $L$ so that $S(1 / 2) \bar{E} \subset S(1)$, where $\bar{E}$ denotes the closure of $E$. Let us prove that the neighborhood $(f ; \bar{V}, E)$ has a compact closure in $D$. Let us put $F=\left\{g \mid g \in D\right.$ and $\left.\left(f^{-1} g\right)(\bar{V}) \subset \bar{E}\right\}$. It clearly suffices to prove that

(A) $I$ is an open mapping in $F$, and

(B) $I(F)$ is closed in $(G \rightarrow L)$.

Proof of (A). Let $g$ be in $F$, and let $\left(g ; C_{1}, E_{1}\right)$ be a given neighborhood of $g$. Let us find a finite subset $\left\{x_{1}, \cdots, x_{k}\right\}$ of $G$ and a positive integer $m$ so that $\left(g ;\left\{x_{1}, \cdots, x_{k}\right\}, S(1 / m)\right) \cap F \subset\left(g ; C_{1}, E_{1}\right)$. For this purpose take a sufficiently large $m$ such that $S(1 / m)^{3} \subset E_{1}$, and take a nucleus $U$ of $G$ such that $U^{m} \subset V$. Since $C_{1}$ is compact we can find a finite subset $\left\{x_{1}, \cdots, x_{k}\right\}$ of $G$ so that $x_{1} U \cup \ldots \cup x_{k} U \supset C_{1}$. We shall prove that $\left\{x_{1}, \cdots, x_{k}\right\}$ and $m$ thus obtained satisfy the requirement.

Let us take an $h$ in $\left(g ;\left\{x_{1}, \cdots, x_{k}\right\}, S(1 / m)\right) \cap F$. Let $x$ be in $C_{1}$. Then $x$ can be written in a form $x=x_{j} y$ where $y \in U$ and $1 \leqq j \leqq k$. Hence $g(x)^{-1} h(x)=\left(g\left(x_{j}\right) g(y)\right)^{-1} h\left(x_{j}\right) h(y)=g(y)^{-1}\left(g\left(x_{j}\right)^{-1} h\left(x_{j}\right)\right) h(y)$. Since $h$ is in $\left(g ;\left\{x_{1}, \cdots, x_{k}\right\}, S(1 / m)\right)$, we have

$$
g\left(x_{j}\right)^{-1} h\left(x_{j}\right) \in S(1 / m) \text {. }
$$

Next for $n=1,2, \cdots, m, y^{n} \in V$ and so $f^{-1}\left(y^{n}\right) g\left(y^{n}\right) \in \bar{E}$, and $f\left(y^{n}\right) \in S(1 / 2)$. Hence $g\left(y^{n}\right) \in S(1 / 2) \bar{E} \subset S(1)$. Because $g\left(y^{n}\right)=g(y)^{n}$ we have $g(y) \in S(1 / m)$ by Proposition 2. Similarly $h(y) \in S(1 / m)$. Hence $g(x)^{-1} h(x) \in S(1 / m)^{3} \subset E_{1}$, namely $h \in\left(g ; C_{1}, E_{1}\right)$.

Proof of (B). Let $\mathrm{Cl}(I(F))$ be the closure of $I(F)$ in $(G \rightarrow L)$. Since $D^{\prime}$ is closed in $(G \rightarrow L)$ we have $\mathrm{Cl}(I(F)) \subset D^{\prime}$. Let $g$ be an element of $\mathrm{Cl}(I(F))$ and let $E_{2}$ be a given nucleus of $L$. We may take $n$ so large that $S(1 / n) \subset E_{2}$. Take a nucleus $W$ of $G$ so that $W^{n+1} \subset V$. Then for an $x$ in $W, x, x^{2}, \cdots, x^{n+1} \in V$ and so $f\left(x^{i}\right)^{-1} h\left(x^{i}\right) \in \bar{E}$ for $h \in F$ and $i=1,2, \cdots, n+1$. Therefore $h\left(x^{i}\right)=h(x)^{i} \in S(1 / 2) \bar{E} \subset S(1)$. Hence by Proposition 2, $h(x) \in S(1 /(n+1))$. Since $g \in \mathrm{Cl}(I(F)), g(x)$ $\in \mathrm{Cl}(S(1 /(n+1))) \subset S(1 / n) \subset E_{2}$, namely $g(W) \subset E_{2}$, which implies that $g \in I(D)$. Thus we proved that $\mathrm{Cl}(I(F)) \subset I(D)$, and so $I(F)$ is closed. 
4. Proof of Theorem 2. Let $G$ be a locally compact group with a compact generating system. Then obviously there is a nucleus $V$ which generates $G$. Let us consider a function $J$ from $D$ into $[\bar{V} \rightarrow L]$ defined by $J f=f \mid \bar{V}$, where $f \mid \bar{V}$ is the restriction of $f$ in $\bar{V}$.

Lemma 1. $J$ is bicontinuous, namely $D$ is homeomorphic with $J(D)$.

Proof. Since $V$ generates $G, J$ is one-to-one. The continuity is obvious. Let us prove the openness of $J$.

Let $\left(f ; C_{1}, E_{1}\right)$ be a given neighborhood of $f$ in $D$. We can find a positive integer $m$ such that $V^{m} \supset C_{1}$. Let us consider a function $\phi$ defined by

$$
\begin{array}{r}
\phi\left(u_{1}, \cdots, u_{m} ; a_{2}, \cdots, a_{m}\right) \\
\quad=a_{m}^{-1}\left(\cdots\left(a_{3}^{-1}\left(\left(a_{2}^{-1} u_{1} a_{2}\right) u_{2}\right) a_{3} u_{3}\right) \cdots\right) a_{m} u_{m},
\end{array}
$$

where $u_{i} \in L$ and $a_{i} \in f(\bar{V})$. Since $\phi\left(1, \cdots, 1 ; a_{2}, \cdots, a_{m}\right)=1$, we can find a nucleus $E_{2}$ of $L$ so that if $u_{1}, \cdots, u_{m}$ are in $E_{2}$, then $\phi\left(u_{1}, \cdots, u_{m}, a_{2}, \cdots, a_{m}\right)$ is in $E_{1}$. Let $x$ be an arbitrary element in $C_{1}$. Then we can find $x_{1}, \cdots, x_{m}$ in $V$ so that $x=x_{1} \cdots x_{m}$. Let $g$ be in $\left(f ; \bar{V}, E_{2}\right)$. Then

$$
\begin{aligned}
f(x)^{-1} g(x) & =\left(f\left(x_{1}\right) \cdots f\left(x_{m}\right)\right)^{-1}\left(g\left(x_{1}\right) \cdots g\left(x_{m}\right)\right) \\
& =\phi\left(u_{1}, \cdots, u_{m} ; a_{2}, \cdots, a_{m}\right)
\end{aligned}
$$

where $f\left(x_{j}\right)^{-1} g\left(x_{j}\right)=u_{j} \in E_{2}$ and $f\left(x_{j}\right)=a_{j} \in f(\bar{V})$. Hence $f(x)^{-1} g(x) \in E_{1}$, namely $\left(f ; \bar{V}, E_{2}\right) \subset\left(f ; C_{1}, E_{1}\right)$, which proves the lemma.

Next let us fix an element $f$ of $D$, and for a $g$ in $D$ we define $g^{*}$ in $[G \rightarrow L]$ by $g^{*}(x)=f(x)^{-1} g(x)$. Then $g^{*}$ satisfies

$$
g^{*}(x y)=f(y)^{-1} g^{*}(x) f(y) g^{*}(y)
$$

for $x$ and $y$ in $G$, and conversely if a function $g^{*}$ in $[G \rightarrow L]$ satisfies (1), then $g^{*}=f^{-1} g$ for some $g$ in $D$. Let $W$ be an arbitrary nucleus of G. Set

$$
F=f^{-1}(f ; \bar{W}, \mathrm{Cl}(S(1 / 2)))=\left\{g^{*} \mid g^{*}(\bar{W}) \subset \mathrm{Cl}(S(1 / 2)) \text { and } g^{*} \in f^{-1} D\right\} \text {. }
$$

Lemma 2. $F \mid \bar{W}=\left\{g^{*}|\bar{W}| g^{*} \in F\right\}$ is an equicontinuous family of functions.

Proof. Let $E$ be a given nuclus of $L$. Let us prove the existence of a nucleus $U$ of $G$ so that $x, x y \in \bar{W}, y \in U$ and $g^{*} \in F$ imply that $g^{*}(x)^{-1} g^{*}(x y) \in E$. For this purpose let us first take an integer $m$, with $S(1 / m) \subset E$, and a positive number $\epsilon$ such that

$$
\mathrm{Cl}(S(1 / 2)) S(\epsilon)^{2} \subset S(1) \text {. }
$$


Next for the $\epsilon$ and the $m$ we shall take a positive number $\delta_{1}$ such that if $a, b \in S(1)$ and $c \in S\left(\delta_{1}\right)$ then

$$
\left(a^{-1} c^{-1} a c b\right)^{j} \in b^{i} S(\epsilon) \quad \text { for } j=1,2, \cdots, m \text {. }
$$

Next let us find a positive number $\delta_{2}$ such that if $a \in S(1)$ and $c \in S\left(\delta_{2}\right)$ then

$$
\left(c^{-j+1} a c^{i-1}\right)\left(c^{-j+2} a c^{j-2}\right) \cdots\left(c^{-1} a c\right) a \in a^{i} S(\epsilon)
$$

for $j=1,2, \cdots, m$, and let $\delta$ be the minimum of $\delta_{1}$ and $\delta_{2}$. Take a nucleus $U_{1}$ of $G$, with $f\left(U_{1}\right) \subset S(\delta)$, and also take a nucleus $U_{2}$ such that $U_{2}^{m} \subset W$, and set $U_{1} \cap U_{2}=U$. Then we have

$$
f(U) \subset S(\delta) \text { and } U^{m} \subset W .
$$

Let $x$ and $x y$ be in $\bar{W}$ so that $y \in U$. Let $g^{*}$ be in $F$. Then $g^{*}(\bar{W})$ $\mathrm{CCl}(S(1 / 2)) \subset S(1)$. By (1) we have

$$
g^{*}(x)^{-1} g^{*}(x y)=g^{*}(x)^{-1} f(y)^{-1} g^{*}(x) f(y) g^{*}(y) .
$$

Hence using (3) we have

$$
\left(g^{*}(x)^{-1} g^{*}(x y)\right)^{i} \in g^{*}(y) i S(\epsilon) \quad \text { for } j=1,2, \cdots, m .
$$

On the other hand, since

$$
g^{*}\left(y^{j}\right)=\left(f(y)^{-j+1} g^{*}(y) f(y)^{j-1}\right) \cdots\left(f(y)^{-1} g^{*}(y) f(y)\right) g^{*}(y),
$$

(4) implies that

$$
g^{*}\left(y^{i}\right) \in g^{*}(y)^{i} S(\epsilon)
$$

From (7) and (8) we have

$$
\left(g^{*}(x)^{-1} g^{*}(x y)\right)^{j} \in g^{*}\left(y^{j}\right) S(\epsilon)^{2} .
$$

From $y^{j} \in W$ it follows that $g^{*}\left(y^{j}\right) \subset \mathrm{Cl}(S(1 / 2))$, whence

$$
\left(g^{*}(x)^{-1} g^{*}(x y)\right)^{i} \in \mathrm{Cl}(S(1 / 2)) S(\epsilon)^{2} \subset S(1)
$$

by (2), for $j=1,2, \cdots, m$. Hence by Proposition 2

$$
g^{*}(x)^{-1} g^{*}(x y) \in S(1 / m) \subset E .
$$

Lemma 3. $F \mid \bar{W}$ is compact, if $\bar{W}$ generates $G$.

Proof. We shall denote by $(\bar{W} \rightarrow \mathrm{Cl}(\mathrm{S}(1 / 2)))$ the topological space composed of all functions from $\bar{W}$ into $\mathrm{Cl}(S(1 / 2))$ with the product topology, and let us consider the identity mapping $I$ from $F \mid \bar{W}$ into $(\bar{W} \rightarrow \mathrm{Cl}(S(1 / 2)))$, which is clearly one-to-one and continuous. Because $(\bar{W} \rightarrow \mathrm{Cl}(S(1 / 2)))$ is compact, it suffices to prove that $I$ is an open mapping and $I(F \mid \bar{W})$ is a closed set. 
(A) Openness of $I$. Let $\mathrm{g}^{*}$ be an element of $F$. For a neighborhood of $g^{*}$ in $F \mid \bar{W}$ we can find a smaller one of the form $\left(g^{*} ; \bar{W}, E\right)$ $=f^{-1}(g ; \bar{W}, E)=\left\{h^{*} \mid h^{*} \in f^{-1} D\right.$ and $\left.\left(g^{*-1} h^{*}\right)(\bar{W}) \subset E\right\}$, where $E$ is a nucleus of $L$. Let us find a finite subset $\left\{x_{1}, \cdots, x_{k}\right\}$ of $\bar{W}$ and a nucleus $E_{1}$ of $L$ such that $\left(g^{*} ; \bar{W}, E\right) \supset\left(g^{*} ;\left\{x_{1}, \cdots, x_{k}\right\}, E_{1}\right) \cap F$, where $\left(g^{*} ;\left\{x_{1}, \cdots, x_{k}\right\}, E_{1}\right)=f^{-1}\left(g ;\left\{x_{1}, \cdots, x_{k}\right\}, E_{1}\right)$.

For this purpose let us first take a nucleus $E_{1}$ of $L$ satisfying $E_{1} a^{-1} E_{1} a E_{1} \subset E$ for $a \in f(\bar{W})$, and find a nucleus $U$ of $G$ in $\bar{W}$ so that $F(U) \subset E_{1}$ using Lemma 2 . Next let us take $x_{1}, \cdots, x_{k} \in \bar{W}$ so that $x_{1} U \cup \ldots \cup x_{k} U \supset \bar{W}$.

Let $x$ be in $\bar{W}$. Then we can find a $y$ in $U$ such that $x=x_{j} y$ for some $j$. Let $h^{*}$ be in $\left(g^{*} ;\left\{x_{1}, \cdots, x_{k}\right\}, E_{1}\right) \cap F$. Then by (1) $g^{*}(x)^{-1} h^{*}(x)$ $=g^{*}\left(x_{j} y\right)^{-1} h^{*}\left(x_{j} y\right)=g^{*}(y)^{-1} f(y)^{-1}\left(g^{*}\left(x_{j}\right)^{-1} h^{*}\left(x_{j}\right)\right) f(y) h^{*}(y)$. On the other hand, since $y \in U$ we have $g^{*}(y) \in E_{1}$ and $h^{*}(y) \in E_{1}$, and $h^{*} \in\left(g^{*} ;\left\{x_{1}, \cdots, x_{k}\right\}, E_{1}\right)$ implies that $g^{*}\left(x_{j}\right)^{-1} h^{*}\left(x_{j}\right) \in E_{1}$. From $U \subset \bar{W}$ it follows that $f(U) \subset f(\bar{W})$. Hence $g^{*}(x)^{-1} h^{*}(x) \subset E$.

(B) $I(F \mid \bar{W})$ is closed.

For a pair $x, y$ of elements of $\bar{W}$ so that $x y$ is also in $\bar{W}$, and for a $g^{*}$ in $F$ we have the relation (1). Hence if $h^{*}$ is in the closure of the image of $F$ in $(\bar{W} \rightarrow \bar{S}(1 / 2))$, then $h^{*}(x y)=f(y)^{-1} h^{*}(x) f(y) h^{*}(y)$. Let us put $h(x)=f(x) h^{*}(x)$ for $x \in \bar{W}$. Then $h(x y)=h(x) h(y)$ for $x, y$, $x y \in \bar{W}$. Since $F \mid \bar{W}$ is equicontinuous by Lemma $2, h^{*}$ is continuous, and so is $h$.

Let $x_{1}, \cdots, x_{m}, y_{1}, \cdots, y_{n}$ be elements of $\bar{W}$. If $x_{1} \cdots x_{m}=y_{1} \cdots$ $y_{n}$, then for $g=f g^{*} \in f F$,

whence we have

$$
g\left(x_{1}\right) \cdots g\left(x_{m}\right)=g\left(y_{1}\right) \cdots g\left(y_{n}\right),
$$

$$
h\left(x_{1}\right) \cdots h\left(x_{m}\right)=h\left(y_{1}\right) \cdots h\left(y_{n}\right) .
$$

Because $\bar{W}$ generates $G, h$ can be extended to a continuous homomorphism $\tilde{h}$ from $G$ into $L$.

Hence $h^{*}=f^{-1} \hbar \in F$, and $h^{*}=h^{*}$ in $\bar{W}$. Therefore $I(F \mid \bar{W})$ is closed.

Proof of Theorem 2. By Lemma $1, D$ is homeomorphic with $J(D)$. On the other hand if we put $W=V$ in Lemma 3 then we have the result that $J(F)$ is compact. Since $[\bar{V} \rightarrow L]$ is a topological group, $J(f) J(F)=J(f ; \bar{V}, \mathrm{Cl}(S(1 / 2))$ is also compact. Accordingly $J(D)$ is locally compact, and so is $D$.

\section{REFERENCES}

1. L. Pontrjagin, Topological groups, Princeton, 1939.

2. A. Weil, L'intégration dans les groupes topologiques et ses applications, Paris, Hermann et Cie., 1940.

\section{University of Pennsyluania}

